\title{
Developmental origins of chronic physical aggression and epigenetics
}

\begin{abstract}
"DNA methylation signatures might, in the future, provide diagnostic and predictive marks of environmentally driven mental health pathologies, such as chronic aggression."
\end{abstract}

\section{KEYWORDS: aggression " development $\approx$ early childhood $\approx$ environment $\approx$ epigenetics " mental health $\approx$ postnatal $\approx$ prediction $\approx$ prenatal $\approx$ prevention}

Over the past half century, two theoretical models strongly influenced research on the development of human physical aggression: social learning and disease onset. According to these developmental perspectives, children learn to physically aggress from their environment, and the onset of the disease is triggered by the accumulated exposure to aggressive models in the environment such as family violence, neighborhood violence and media violence. Most of the evidence came from studies of school-aged children and adolescents. Recent longitudinal studies tracing developmental trajectories of physical aggression from early childhood onwards suggest an inversed developmental process. Frequency of physical aggression is at its peak during early childhood and, as they age, children learn socially acceptable behaviors from interactions with their environment $[1,2]$. A 'disease' status is eventually given to children who failed to learn socially acceptable behaviors. The mechanisms that lead to deficits in learning to use socially accepted behaviors appear to be strongly intergenerational, based on complex genetic and environmental contributions.

Longitudinal studies initiated during the perinatal period suggest that several early life environmental factors are involved in triggering the mechanisms that lead to deficits in learning to use socially acceptable behaviors for solving problems. These include family characteristics (e.g., poverty and marital conflicts), maternal history of behavior problems (e.g., antisocial behavior, school failure, teen pregnancy and depression), maternal lifestyle during pregnancy (e.g., smoking) and maternal parenting practices (e.g., coercive discipline) [2]. These family and maternal characteristics can be used to identify at-risk pregnant women and offer them adequate support [3].
Genetic studies have also identified sequence differences in critical genes such as $M O A$, the serotonin transporter and others, which may play a role in the development and maintenance of chronic aggression. However, an increasing number of studies are demonstrating that phenotypic variations in behavior cannot be accounted for by just these genetic differences, and that the phenotype would best be accounted for by an interaction of genetic and environmental factors. Experiments with nonhuman primates that measured the impact of maternal deprivation on the behavior of monkeys with different 5-HTT genotypes illustrated how gene-environment interactions might provide a better explanation for certain behavioral phenotypes such as aggression than genetic differences per se $[4,5]$. These data suggest that social-environmental triggers are extremely important in defining the final phenotype and they can override a phenotype driven by sequence differences present in critical genes. This primate study is consistent with other studies in humans. Association studies in humans have suggested, for example, that violent behavior in humans was more frequent for males who were maltreated during childhood when they had a genotype conferring low MAO-A activity [6]. However, further studies indicate that these gene-environment statistical interactions may depend on age, severity of maltreatment and situational factors, and may apply to many other types of behavior problems [7-9].

A criticism of the approach to mental health that focuses on statistical interactions between one gene variant and one environmental factor is that it ignores the potential diverse statistical and bio-psycho-social interactions between multiple gene variants and varied environmental conditions that lead to a multitude of endophenotypes and phenotypes related to mental

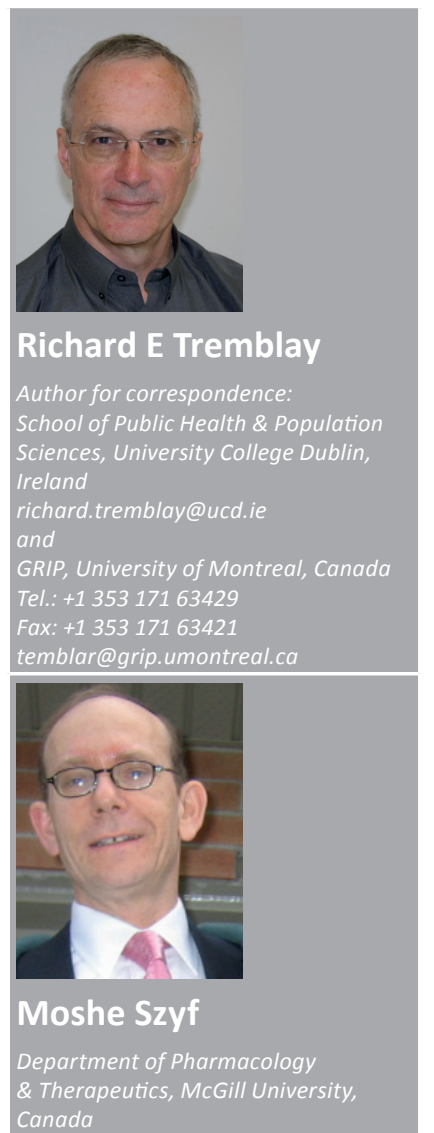

future medicine $^{\text {pits }} \mathbf{f S g}$ 
health problems [10]. The realization that environmental and genetic variants interact was an important step forward towards understanding the mechanisms which underpin the development of mental health problems. However, it is clear that our challenge is to involve a more comprehensive understanding of the relationship between the environment and the genome in mental health.

How do we define an individual's environment? The common simplistic approach that provides an overall description of an individual's environment spanning over a 15 - to 20 -year period as having been maltreated or not, is obviously inadequate from a developmental perspective [11]. We propose that it is critical to take a developmental perspective when defining environment. Complex disorders such as mental illnesses are most probably based on interactions of several gene variants and several environmental factors at different time points during development. It is essential to take into account the critical developmental periods of the different disorders. Pregnancy and early childhood time points should clearly be the focus for understanding gene-environment effects on the development of chronic physical aggression.

\section{“...early life adversity has a long-term impact on physical health and mental health independent of the adversity situation in adulthood."}

What are the mechanisms behind the elusive term 'gene-environment' interactions? The common understanding is that the term refers to modulation, either attenuation or exacerbation, of the effect of a gene variant on phenotype by an environmental exposure. To date, this has been to date a statistical concept and the interaction was rarely dissected from a mechanistic point of view. However, it is possible to look at this relationship mechanistically as an interaction between environmental signals and long-term programming of gene expression, irrespective of whether the genes are variants or not. In this mechanistic context it would be possible to weigh up whether variants could affect the programming of gene expression by the environment, and thus, explain why certain gene variants have a stronger impact on the phenotype than the common allele. The question that should be posed, therefore, is; how does the environment program longterm gene-expression profiles? What are the mechanisms that maintain the memory of the early environmental exposure in the genome long after that exposure is gone? For example, early life adversity has a long-term impact on physical health and mental health independent of the adversity situation in adulthood. How does the genome memorize behavioral environmental exposures?

A total of 4 years after Darwin published On the Origin of Species (1859) [12], the French naturalist Quatrefages (1863) wrote [13]:

"Nowadays I admit, with everybody, the doctrine of epigenesis. Every normal egg which gives birth to an abnormal individual is influenced by external agents whatever they are; this is what I call action of the milieu."

A century and a half later, we are starting to understand the pathways leading from environmental exposures to the programming of gene expression that can create a stable and lasting change in gene function without modifying its sequence. The mystery of epigenesis has been partly unraveled in the last three decades. The genome is programmed during gestation to perform its differential functions in the different tissues of the body by the epigenome, which consists of chromatin modification and the positioning of nucleosomes along the gene regulatory sequences, noncoding RNAs, as well as the chemical modification of the DNA itself by methylation mainly at the CG dinucleotide. Different cell types exhibit different patterns of methylation at the CG dinucleotides in their genomes [14]. Several lines of evidence have shown that DNA methylation at the 5' regulatory regions of genes silences their transcription [15]; however, recent whole-genome methylation mapping might suggest that DNA methylation in the bodies of genes plays a positive role in gene expression [16]. It is possible that DNA methylation plays regulatory roles in genome function that are beyond our current understanding of the silencing of coding mRNA transcription. DNA methylation is an extremely stable mark that is copied by dedicated enzymes called DNA methyltransferases. The extreme chemical stability of the DNA methylation mark, the fact that it is part of the chemistry of the DNA molecule and the fact that there is an enzymatic process that can copy the DNA methylation mark positions it as a prime bearer of the epigenetic memory. Although it was originally believed that DNA methylation is fixed after birth and terminal differentiation of cells, recent data demonstrates that DNA methylation could change during the course of life, and 
probably does so in response to environmental triggers. Remarkably, the maternal care environment early in life modulates DNA methylation and other epigenetic marks, and this modulation remains stable later in life [17]. If this is indeed the case, then DNA methylation might be a mechanism through which the 'social environment' interacts with 'genes'. Our studies with the maternal care model also provided the first clue of how this seemingly mysterious ephemeral social environment and the hardcore chemistry of DNA interact. Maternal care elicits a serotonergic firing in the brain that triggers a cAMP signaling pathway that activates a transcription factor, NGFIA. This transcription factor interacts with its targets in the genome and recruits a histone acetyl transferase CBP and a DNA modulating protein MBD2 to its targets [18]. There are, therefore, potential conduits between the social environment and the genome that are highly active early in development and could target specific changes in DNA methylation and chromatin modification in critical tissues, including the brain. Thus, environments can impact phenotypes through their biochemical impact on programming of gene function $[19,20]$.

\section{"Epigenetic studies are potentially more likely to unravel the actual physiological interactions because they focus specifically on the biochemical effects of the environment on gene expression at a given moment in time."}

In addition to its value in explaining mechanisms of environment-gene interactions, DNA methylation is a superb diagnostic mark because of its chemical stability and the possibility of using the extensive methodology being developed for genomic research to map DNA methylation variations. DNA methylation signatures might, in the future, provide diagnostic and predictive marks of environmentally driven mental health pathologies, such as chronic aggression. DNA methylation and other epigenetic marks are potentially reversible by either pharmacological, or perhaps, even behavioral interventions. Therefore, in addition, to diagnostic aspects of this new understanding of 'gene-environment' interactions, the possibility that DNA methylation and other epigenetic factors play a role in defining the phenotype raises the hope of pharmacological and behavioral interventions that will reverse these epigenetic marks. Epigenetic effects and their immense diagnostic and therapeutic potential are well known in cancer research [21], and have recently been suggested to play an important role in the obesity epidemic we are facing [22]. The possibility that they might be driving behavior regulation is a paradigm shift in the mental health field $[20,23]$.

To grasp the potential contribution of epigenetics to our understanding of the mechanisms involved in early development of chronic physical aggression, it is useful to define the difference between the traditional gene-environment interaction theory and the epigenetic theory. To elucidate the key differences we can use two recent studies which attempted to explain the effects of a maltreating environment on behavior development. In a classic gene-environment statistical interaction study [2], males brought up in a 'maltreating' environment were observed to be more at risk of violent behavior if they had a short rather than a long allele on the promoter region of the $M A O A$ gene. In this study, the maltreating environment is not shown to physiologically interact with the $M A O A$ gene. The interaction is statistical, (i.e., obtained from a $2 \times 2$ table comparing four groups created from two dichotomous variables: maltreating-not maltreating and short-long $M A O A$ allele). It is presumed that individuals who inherited either short or long alleles react differently to an environment that was maltreating because their neural system functions differently and such differences are due to $M A O A$ activity. The developmental timing of the presumed gene-environment interaction is unspecified.

Epigenetic studies are potentially more likely to unravel the actual physiological interactions because they focus specifically on the biochemical effects of the environment on gene expression at a given moment in time. It will be possible to delineate the signaling pathways leading from the environmental trigger to the change in DNA and to then study the implication of these changes on gene expression. The classic example of the effects of a maltreating environment comes from an experimental study of maternal behavior in rats which showed that rat pups insufficiently licked by their mothers in the days following birth (i.e., neglected) exhibit increased methylation of the gene encoding the glucocorticoid receptor in the hippocampus, resulting in reduced expression of the gene [17,24]. The study further showed that this gene methylation effect had downstream effects on the hypothalamic-pituitary-adrenal axis, which regulates stress responses in the body. More recently, a study of maternal separation at birth with mice showed DNA methylation alterations associated with chronic hypersecretion of corticosterone, problems with stress coping and memory in the adult offspring [25]. 
Epigenetic mechanisms are especially important because they provide a powerful explanation for maternal transmission of behavior disorders that extend beyond the traditional genetic transmission explanations. The mother, through her maternal behavior, can affect DNA methylation of critical genes in the offspring [26]. Furthermore, DNA methylation changes over time can be used as markers of environmental effects of gene expression during development, including the assessment of various preventive and corrective intervention effects such as changes in lifestyle during pregnancy (e.g., diet, stress and smoking) or the improvement of parenting practices.

As discussed earlier, it is unlikely that the environment will affect a single gene or that a single component in the environment will act in isolation to modulate the phenotype. The emergence of whole-genome DNA methylation mapping methodologies allows us to map the responses of a combination of functional gene circuitries to environmental triggers at different developmental time points. By identifying the genomic targets of environmental triggers it might be possible to generate hypotheses on the physiological pathways involved. It stands to reason that the responses to social environment early in life are not limited to the brain, and that peripheral tissues are affected as well. Thus, although DNA methylation patterns are partly tissue specific and it is impossible to access the brain for DNA methylation analyses of longitudinal studies in humans, peripheral tissues such as a specific subset of white blood cells might be good candidates. However, this remains to be seen. Several studies are ongoing to test this hypothesis.

Longitudinal studies of chronic physical aggression development indicate that early risk factors are specifically related to maternal characteristics (e.g., maternal age at first pregnancy, history of behavior problems, education, smoking, depression and coercive parenting). This can easily be understood from the traditional environmental perspective: a poor early environment has an impact on the developing fetus and infant. Mother characteristics turn out to be more important risk factors than father characteristics because the former carry the child in their womb during fetal life and are more involved in caregiving during early childhood. However, the exact bio-psycho-social mechanisms linking poor quality environment to disorganized behavior remains unclear, to say the least.
Epigenetics provides a basic mechanism that is parsimonious, testable and promising for understanding and preventing behavior problems. The most fascinating aspect of this mechanism is that it provides an environmentally based explanation of intergenerational transmission for physical and mental disorders, which involves genes but is not genetically transmitted. These mechanisms are still far from being clearly understood, but they provide a challenging alternative perspective to the traditional gene versus environment and gene-environment interaction hypotheses. Although the original epigenetic effects on behavior development used rats [17], recent studies with humans suggest that environments have similar effects on human behavior development. In a study of brains from individuals who committed suicide, epigenetic differences were observed in a neuron-specific glucocorticoid receptor promoter when three groups were compared: significant differences in methylation was observed when the brains of those who committed suicide and had been abused during childhood were compared with the brains of those who were not abused, and those of a group that had died in car accidents [27]. A pilot study [20] is also indicating differences in methylation profiles from blood samples of males on chronic and normal trajectories of physical aggression. Our preliminary studies indicate clear differences in DNA methylation profiles between the aggressive and nonaggressive groups [PROvENÇAL et AL., Unpublished Data].

\section{"Epigenetics provides a basic mechanism that is parsimonious, testable and promising for understanding and preventing behavior problems."}

A key hypothesis from crossfostering and epigenetic studies with a bearing on the idea of early and intergenerational prevention is that environmental effects are transmitted intergenerationally, and most clearly from mothers to daughters [28-31]. In its simplest form it is easy to understand that if maternal lifestyle (e.g., eating, drinking, smoking, stress and depression) during pregnancy has an epigenetic effect on the daughter's brain development and functioning, the daughter who is likely to live in a environment similar to her mother's will have a similar lifestyle during her own pregnancies, and so on from generation to generation [32]. From this perspective, mother to daughter epigenetic transmission of behavior problems would be the hidden mechanism of intergenerational transmission of male chronic aggression. 


\section{Financial \& competing interests disclosure}

The authors have no relevant affiliations or financial involvement with any organization or entity with a financial interest in or financial conflict with the subject matter or materials discussed in the manuscript. This includes employment, consultancies, honoraria, stock ownership or options, expert testimony, grants or patents received or pending, or royalties.

No writing assistance was utilized in the production of this manuscript.

\section{Bibliography}

1 Côté SM, Boivin M, Nagin DS et al:: The role of maternal education and non-maternal care services in the prevention of children's physical aggression. Arch. Gen. Psychiatr. 64(11), 1305-1312 (2007).

2 Tremblay RE: Developmental origins of disruptive behaviour problems: the 'original sin' hypothesis, epigenetics and their consequences for prevention. J. Child Psychol. Psychiatr. 51(4), 341-367 (2010).

3 Eckenrode J, Campa M, Luckey DW et al.: Long-term effects of prenatal and infancy nurse home visitation on the life course of youths: 19-year follow-up of a randomized trial. Arch. Pediatr. Adolesc. Med. 164(1), 9-15 (2010).

4 Bennett AJ, Lesch KP, Heils A et al.: Early experience and serotonin transporter gene variation interact to influence primate CNS function. Mol. Psychiatr. 7(1), 118-122 (2002).

5 Champoux M, Bennett A, Shannon C, Higley JD, Lesch KP, Suomi SJ: Serotonin transporter gene polymorphism, differential early rearing, and behavior in rhesus monkey neonates. Mol. Psychiatr. 7(10), 1058-1063 (2002).

6 Caspi A, McClay J, Moffitt T et al.: Role of genotype in the cycle of violence in maltreated children. Science 297, 851-854 (2002).

7 McDermott R, Tingley D, Cowden J, Frazzetto G, Johnson DD: Monoamine oxidase A gene $(M A O A)$ predicts behavioral aggression following provocation. Proc. Natl Acad. Sci. USA 106(7), 2118-2123 (2009).

8 Tikkanen R, Sjoberg RL, Ducci F et al.: Effects of $M A O A$-genotype, alcohol consumption, and aging on violent behavior. Alcohol. Clin. Exp. Res. 33(3), 428-434 (2009).
9 Weder N, Yang BZ, Douglas-Palumberi $\mathrm{H}$ et al.: $M A O A$ genotype, maltreatment, and aggressive behavior: the changing impact of genotype at varying levels of trauma. Biol. Psychiatr. 65(5), 417-424 (2009).

10 Risch N, Herrell R, Lehner T et al.: Interaction between the serotonin transporter gene (5-HTTLPR), stressful life events, and risk of depression a meta-analysis. J. Am. Med. Assoc. 301(23), 2462-2471 (2009).

11 van der Vegt EJM, Oostra BA, Arias-Vasquez A, van der Ende J, Verhulst FC, Tiemeier H: High activity of monoamine oxidase $\mathrm{A}$ is associated with externalizing behaviour in maltreated and nonmaltreated adoptees. Psychiatr. Genet. 19(4), 209-211 (2009).

12 Darwin C: On the Origin of Species. John Murray, London, UK (1859).

13 Quatrefages A: Débats du 16 juillet 1863 de la Société d'Anthropologie de Paris. Bull. Mem. Soc. Anthropol. Paris 4, 378-383 (1863).

14 Razin A, Riggs AD: DNA methylation and gene-function. Science 210 (4470), 604-610 (1980).

15 Stein R, Razin A, Cedar H: In vitro methylation of the hamster adenine phosphoribosyltransferase gene inhibits its expression in mouse L-cells. Proc. Natl Acad. Sci. USA 79(11), 3418-3422 (1982).

16 Hellman A, Chess A: Gene body-specific methylation on the active $\mathrm{X}$ chromosome. Science 315(5815), 1141-1143 (2007).

17 Weaver ICG, Cervoni N, Champagne FA et al.: Epigenetic programming by maternal behavior. Nat. Neurosci. 7(8), 847-854 (2004).

18 Weaver ICG, D'Alessio AC, Brown SE et al:: The transcription factor nerve growth factor-inducible protein A mediates epigenetic programming: altering epigenetic marks by immediate-early genes. J. Neurosci. 27(7), 1756-1768 (2007).

19 Mill J, Petronis A: Pre- and peri-natal environmental risks for attention-deficit hyperactivity disorder (ADHD): the potential role of epigenetic processes in mediating susceptibility. J. Child Psychol. Psychiatr. 49(10), 1020-1030 (2008).

20 Szyf M, Weaver ICG, Provençal N, McGowan P, Tremblay RE, Meaney MJ: Epigenetics and behaviour. In: Development and Prevention of Behaviour problems: from Genes to Social Policy. Tremblay RE, van Aken MAG, Koops W (Eds). Psychology Press, Sussex, UK 25-59 (2009).
21 Szyf M: Targeting DNA methylation in cancer. Ageing Res. Rev. 2(3), 299-328 (2003).

22 Waterland RA: Is epigenetics an important link between early life events and adult disease? Horm. Res. 71(Suppl. 1), 13-16 (2009).

23 Meaney MJ, Szyf M: Maternal care as a model for experience-dependent chromatin plasticity? Trends Neurosci. 28(9), 456-463 (2005).

24 Weaver ICG, Meaney MJ, Szyf M: Maternal care effects on the hippocampal transcriptome and anxiety-mediated behaviors in the offspring that are reversible in adulthood. Proc. Natl Acad. Sci. USA 103(9), 3480-3485 (2006).

25 Murgatroyd C, Patchev AV, Wu Y et al: Dynamic DNA methylation programs persistent adverse effects of early-life stress. Nat. Neurosci. 12(12), 1559-1566 (2009).

26 Champagne FA, Weaver ICG, Diorio J, Dymov S, Szyf M, Meaney MJ: Maternal care associated with methylation of the estrogen receptor- $\alpha 1 \mathrm{~b}$ promoter and estrogen receptor- $\alpha$ expression in the medial preoptic area of female offspring. Endocrinology 147(6), 2909-2915 (2006).

27 McGowan PO, Sasaki A, D’Alessio AC et al.: Epigenetic regulation of the glucocorticoid receptor in human brain associates with childhood abuse. Nat. Neurosci. 12(3), 342-348 (2009).

28 Champagne F, Meaney MJ: Like mother, like daughter: evidence for non-genomic transmission of parental behavior and stress responsivity. Progr. Brain Res. 133, 287-302 (2001).

29 Diamond A: The interplay of biology and the environment broadly defined. Develop. Psychol. 45(1), 1-8 (2009).

30 Fish EW, Shahrokh D, Bagot R et al: Epigenetic programming of stress responses through variations in maternal care. Ann. NY Acad. Sci. 1036, 167-180 (2004).

31 Meaney MJ: Maternal care, gene expression, and the transmission of individual differences in stress reactivity across generations. Ann. Rev. Neurosci. 24, 1161-1192 (2001).

32 Gluckman PD, Hanson MA, Bateson P et al:: Towards a new developmental synthesis: adaptive developmental plasticity and human disease. Lancet 373(9675), 1654-1657 (2009). 